\title{
Encapsulation of anticancer drug doxorubicin inside dendritic macromolecular cavities: first-principles benchmarks
}

\author{
Hatem Besrour, Bahoueddine Tangour \\ Université de Tunis Al Manar, Research Unit of Modeling in Fundamental Sciences and Didactic \\ Team of Theoretical Chemistry, Tunis 1060, Tunisia. \\ Roberto Linguerri, Majdi Hochlaf* \\ Université Paris-Est, Laboratoire Modélisation et Simulation Multi Echelle, MSME UMR 8208 \\ CNRS, 5 bd Descartes, 77454 Marne-la-Vallée, France.
}

Keywords: ab initio, dendrimers, encapsulation, anticancer drug.

*author for correspondence:

M. Hochlaf: phone: +33 1609573 19. FAX: +33 1609573 20. Email: hochlaf@univ-mlv.fr 


\begin{abstract}
By using first-principles approaches based on Density Functional Theory, we explore the possibility of using dendritic macromolecular structures as carriers of the doxorubicin anticancer drug. In particular, we consider macromolecular cavities of different sizes composed of phenylene-, thiophene-, phenyl-cored thiophen- and thioazole-based dendrimers. The comparison between the optimized molecular geometries of the monomers and of the host-guest complexes reveals that only slight structural changes are observed in doxorubicin upon complexation. Also, the encapsulation energies for the host-guest complexes suggest that these systems are of potential use for pharmacology applications in vivo. The interaction of the guest doxorubicin with the macromolecular cavities exploits different types of weak intermolecular forces including $\sigma, \pi$ and hydrogen bond interactions. The electronic structure of these complexes is discussed, with particular emphasis placed on the role of the charge distribution and the nature of the frontier molecular orbitals in the encapsulation process. Spectroscopic properties of these complexes are derived to facilitate their detection in laboratory and in vivo. These include IR vibrational frequencies, absorption wavelengths and relative oscillator strengths for the main transitions in the UV-Vis spectrum.
\end{abstract}




\section{Introduction}

Cavity-containing supramolecular structures represent an important branch of supramolecular chemistry [1]. Among the reasons is the high tunability of the cavity size and shape that can be obtained by varying the nature of the constituting monomers and of their sequencing [2]. Cavities inside macromolecular structures can be rigid, like in cyclodextrins or cucurbiturils, or flexible, like in calixarenes, as a result of the complex pattern of intra- and inter-molecular interactions. In particular, non-rigid structures with readily functionalized end-groups are versatile systems for host-guest chemistry applications, especially in the bio-medical field, where new and effective classes of active compounds with antiviral, bactericidal and anticancer activities can be prepared [3].

Falling in this category, dendrimers, or arborols, are a special class of flexible polymeric molecules with unique properties and structures. While linear standard polymers are synthesized from $\mathrm{XY}$ monomeric units, where $\mathrm{X}$ and $\mathrm{Y}$ represent molecular moieties with mutual complementary binding properties, dendrimers are the result of the polymerization of $\mathrm{XY}_{n}$ units, with $n$ typically ranging between 2 and 3. This leads to a high degree of branching (hyperbranching) during the growing steps of the polymeric entity. The dendrimer synthesis is typically started (divergent synthesis) from a central molecular core and performed in a multistep fashion, resulting in an expanding globular, radially symmetric and multishell macromolecular architecture, where the number of monomers included in each shell is doubled or tripled at each iteration, depending on the value of $n$. The number of layers in a growing dendrimer corresponds to the generation number $\mathrm{G} n$ (the core being the generation zero, $\mathrm{G} 0$, dendrimer), which can be easily inferred by counting the number of branching points (focal points) encountered when going from the dendrimer core to its surface. Since the first reported synthesis of organic dendrimers by Vögtle in 1978 [4], several dendritic structures have been produced, some of them as commercially available compounds, like the PAMAM (poly(amido amine)), developed by Tomalia's group at Dow Chemicals [5,6], and the PMI (poly (propylene imine)).

Among the interesting properties of these macromolecular structures are the monodispersity, at least for sub-nano scaled dendrimers, and the possibility of functionalising the end-groups at the exposed surface of the dendrimer outer shell with different groups, thus inducing specific physical and chemical properties that can be tuned for applications in supramolecular and biological chemistry. Dendrimers conformational properties depend to a large extent on the flexibility of the dendrons and on the capacity of the end-groups at the outer shell to interact with each other, e.g. by hydrogen bonding, thus creating dense structures.

Back to 1983, molecular simulations by De Gennes and Hervet [7] showed that dendrimers present rather dense peripheral regions and relatively empty zones close to the core, where molecules of variable size can be trapped in cavities, the volume of which can be large enough to accommodate rather big molecules [8]. Inside the dendrimers, dendrons are characterised by high mobility allowing the 
cavities to be remodelled according to the interactions with the guest molecule, either by van der Waals / electrostatic forces or by hydrogen bonding. The quite dense outer shell of the dendrimers has the advantage of isolating the guest molecule from the solvent. Therefore, dendrimers can be potentially used as molecular containers (dendritic boxes) for drug delivery purposes. Other factors like solvent effects, ionic strength and $\mathrm{pH}$ can modify the dendritic structure, where a competition between expanded molecular conformations or more compact globular structures, with extensive dendron back-folding, may occur [9]. The encapsulation of one or more guest-molecules inside the dendrimer cavities further complicates the picture. Nowadays, the study of these conformational changes can be effectively performed by molecular dynamics simulations using force fields or by first-principles methodologies.

At the microscopic scale, the nature of the interactions between the guest and the host molecules depends on their chemical composition, local structure and on the solvent. Encapsulation of a guest entity inside the cavity of a macromolecular system will depend on the interplay between dispersion forces, electrostatic interactions and hydrogen bonding. Due to the complexity of these systems, predictions of encapsulation energies, final structures, and elucidation of the nature of intermolecular forces between the host and guest molecule require advanced all-atom or coarse-grained molecular dynamics approaches based on carefully tailored force fields. [10-12]

One guest molecule that could be proficiently used in the context of drug delivery to enhance its biological activity and possibly minimize the side effects is the popular pharmaceutical doxorubicin (DXR). Doxorubicin, an anthracycline antibiotic, is a very effective anti-tumor agent with a broad spectrum of applications. It is used to treat several human neoplasms, including acute leukemia, lymphoma, stomach, breast, ovarian and bone cancers, though causing cardiotoxicity at the same time. Its mechanism of action is based on the reactivity between the anthracycline chromophore and the DNA base pairs of the target cell, resulting in DNA intercalation, interruption of DNA replication and eventually death of the tumor cell [13-15]. To assess the possibility of using these macromolecular carriers for pharmacology applications, thorough investigations of their structural, chemical and thermodynamical properties as well as a full characterization of their spectra are needed. In particular, non-invasive spectroscopic techniques are useful for fast and unambiguous identifications of these species, both in the laboratory and in vivo, and their precise monitoring.

In this contribution, we report encapsulation energies, optimized geometries, charge distributions, IR vibrational frequencies, absorption wavelengths and relative oscillator strengths for the main transitions in the UV-Vis spectrum of the DXR molecule trapped in phenylene- $(\mathrm{P})$, thiophene(T), phenyl-cored thiophen- (TP) and thioazole-based (TA) dendrimers. These calculations are carried out with advanced first-principles methodologies based on appropriate exchange-correlation functionals and atomic basis functions, where suitable corrections accounting for dispersion forces have been added to the calculated energies. Specifically, we show that encapsulation is governed by both $\pi-\pi$ stacking 
and $\sigma$-type interactions, with some contributions from hydrogen bonding as well. This leads to specific cage forms where the aromatic cycles of the guest and host molecules are found either in parallel stacking configurations or in geometric arrangements that maximize host-guest overlap. Given the size of the cavities considered in this study we focused only on the 1:1 stoichiometry for the complexation process, since larger guest-host ratios should be unlikely.

\section{Computational method}

Electronic structure calculations have been carried out with the GAUSSIAN 09 [16] suite of programs using the density functional theory (DFT) in conjunction with the non-empirical PBE [17] and semi-empirical B3LYP [18,19] exchange-correlation functionals, and the 6-31G [20,21] and 6-31G (2df,p) [22] atomic basis sets. The 6-31G and 6-31G (2df,p) bases differ by the addition of two sets of $p$ functions to the hydrogen atom and two sets of $d$ and one set of $f$ functions to the first and second row elements. The higher angular momentum functions are generally needed for a better description of the long-range interactions that involve a certain degree of atomic polarization. The calculation of encapsulation energies for the guest molecules trapped inside the molecular cavities requires accurate evaluation of the dispersion interactions, which were not explicitly considered in the construction of the above-cited functionals. The inclusion of these effects, mandatory for the accurate prediction of encapsulation geometries and energies, was achieved by adding to the calculated DFT energies the empirical D3 dispersion correction [23], as implemented in GAUSSIAN. This correction is known for its favourable accuracy/cost ratio and is particularly suited to treat dispersion forces in large molecular aggregates [24].

The choice of the exchange-correlation functionals employed in this study is justified by the following considerations: i) PBE is a non-empirical functional based on the generalized gradient approximation that proved to be of general applicability to a large variety of systems, from isolated molecules or complexes in the gas phase, to bulk metals and organic reactions involving metal surfaces. The universal character of this functional is well suited for treating molecular systems in a variety of complex chemical environments, like the cavities of macromolecular structures. ii) Compared to other popular non-empirical functionals, PBE displays a similar, and in some cases better, accuracy with respect to TPSS and PW91 in the evaluation of hydrogen bond and dispersion energies [25-27]. iii) Although nonempirical functionals like PBE have the tendency to slightly overestimate absolute bond lengths, they give much more accurate results for relative quantities like energy differences and bond length changes. This property is shared by B3LYP hybrid functional as well, largely due to extensive error cancellation. iv) Among the plethora of available DFT functionals, B3LYP is one of the most popular. It shows good performance, especially when applied to organic molecules, for the prediction of energies, structures and properties. Even if simple B3LYP is well known for providing poor 
description of dispersion and long range interactions [28], B3LYP-D3 is capable of reproducing binding energies of hydrogen-bonded dimers to an accuracy of better than $0.5 \mathrm{kcal} \mathrm{mol}^{-1}$ [29].

To reduce the computational cost of these computations to a manageable level, model cages were adopted, where the branches of the dendritic structures pointing out of the macromolecular cages were substituted with hydrogen atoms and thus excluded from these relaxations. The adopted models are large enough to be considered as realistic representations of the encapsulated drug inside the dendritic framework and should lead to accurate evaluations of the energetic and structural encapsulation properties for these systems.

In the molecular geometry optimizations, several initial arrangements for the host-guest complex were considered, corresponding to both inclusion and exclusion complex geometries. These structures, which include also partial encapsulation of the guest entity, where pre-optimized by a simple molecular mechanic (MM) approach based on the general AMBER force field (GAFF) [30]. A full atomic relaxation of the host cavity interacting with the guest molecule was then performed at the DFT level. In the following sections, only the most stable complexes, as proved by the DFT calculations, are presented. The stationary points corresponding to stable isomers (i.e. local minima on the potential energy surface) were characterised by inspection of the harmonic vibrational frequencies (all positive) obtained from standard normal mode analysis. Encapsulation energies were evaluated by subtracting the sum of the electronic energies of the isolated host and guest units (fully relaxed) from the energy of the complex. No corrections for basis set superposition error were applied. To gain insight into the charge distribution of the guest-host complexes we performed a natural bond orbital (NBO) analysis [31] as implemented in GAUSSIAN 09. The computations of the electronic transitions in the UV-Vis spectra for the isolated and complexed chromophores have been performed by means of the time-dependent density-functional theory (TD-DFT) [32-36] at the B3LYP level. The good performance of this approach for the calculation of singlet-singlet vertical excitation energies, both in terms of accuracy and computational efficiency, is well-established (see Refs. [37,38] for instance), providing that no appreciable charge transfer occurs in the excitation process and the monodeterminantal picture is a good zero-order approximation of the electronic wavefunction. Previously, we showed, through comparisons with experiments and to reference post Hartree-Fock methods, that the present theoretical approach is accurate enough to derive the desired properties of this type of complexes [39-44].

\section{Results and discussion}

\section{a. Structures of isolated DXR, cavities and encapsulated DXR}

Figure 1 displays the optimized structure of DXR (guest), obtained at the PBE+D3 / 6-31G (2df,p) level of theory, along with the adopted atom-labelling scheme. This corresponds to the most 
stable conformer that could be identified in our molecular structure optimizations. We also show the hydrogen bonds contributing to the stabilization of this conformer via the formation of 5-6 rings. In addition, Figure 1 gives also the 3D molecular electrostatic potential (3D MEP) mapped over the groundstate electronic isodensity surface. This picture reveals the electron-rich and electron-poor regions of the DXR unit that could interact with different domains in the host cavities and possibly favor the stabilisation of the host-guest complexes. In particular, we remark negative electrostatic potentials (red regions) in close proximity to the oxygen atoms, notably $\mathrm{O} 11$ in the accessible $\mathrm{O} 11-\mathrm{H} 28$ hydroxyl group and $\mathrm{O} 1, \mathrm{O} 3$ and $\mathrm{O5}$. On the other hand, positive electrostatic potentials (blue regions) are localized in the vicinity of the amino and methyl groups, as expected.

We report in Table 1 some selected bond lengths and dihedral angles for the DXR molecule, calculated with B3LYP+D3 and PBE+D3 and the two basis sets employed in this study. These data show rather good agreement of the structural parameters calculated with these two approaches. In fact, for bond lengths we compute mean absolute differences between B3LYP and PBE of 0.0113 and 0.0119 $\AA$ when the $6-31 \mathrm{G}$ and $6-31 \mathrm{G}$ (2df,p) basis sets are used, respectively, with maximum absolute differences of $0.0350 \AA$ for both basis sets. The basis set effect on bond lengths is slightly more pronounced: we obtain mean absolute differences between the 6-31G and 6-31G (2df,p) sets of 0.0249 and $0.0245 \AA$ when B3LYP+D3 and PBE+D3 are adopted, respectively, with maximum absolute differences of 0.0390 and $0.0400 \AA$, in the same order. In particular, rather large deviations are observed for the $\mathrm{CO}$ distances, whereas somehow smaller discrepancies are computed for the other bond lengths. Similar trends are observed for the dihedral angles as well. Indeed, the chosen method has almost no influence on the calculated values when the 6-31G(2df,p) basis set is used, while a small mean absolute difference of around 1.3 degrees is observed for the smaller basis set. The basis set effect is again stronger: mean absolute differences in the values calculated with the two basis sets are roughly 4 and 3 degrees for the B3LYP+D3 and PBE+D3 methods, respectively. In sum, we note that bond lengths almost systematically decrease upon extension of the basis set for a given method and when going, for a given basis set, from PBE to B3LYP. The observed basis set effect on bond lengths is expected because of the better description of electron correlation obtained with the larger 6-31 G(2df,p) set compared to 6-31G. Thus, the structural analysis is quite independent on the selected functional, while a slight effect is observed upon extension of the atomic basis set. Similar trends are expected for the larger systems considered in this study. Following this analysis, the PBE+D3 / 6-31 G(2df,p) level is the best suited for the description of the larger molecular systems under investigation.

Figure 2 displays the host cavities based on P, T, TP and TA units selected in our simulations, as optimized at the PBE+D3 / 6-31G (2df,p) level of theory, together with the generating monomers and atom labelling. The structures of the monomers represented in the left-hand side of Figure 2 have been optimised at the same level of theory as the cavities after attaching hydrogen atoms at the branching 
points. For each dendrimer type, we considered two model cavities of different sizes, i.e. a small one (S) and a large one (L), both depicted in the right-hand side of Figure 2. Small and large cavities are composed of between three and five monomeric units. These model cavities, which do not include the peripheral branches of the complete dendritic structure in the aim of simplifying the theoretical treatment, have been chosen because they represent major recurring motifs inside the respective dendrimers $[45,46]$ and, given their size, can easily accommodate the guest molecule. They are not rigid and possess high torsional flexibility as reflected by the small harmonic vibrational frequencies / force constants calculated for the ring deformation modes. Nevertheless, they exhibit several close to planar structural parts to favour the electron delocalisation over the monomeric aromatic cycles, which contribute to their stabilisation.

Table 2 reports the dimensions of the different cavities where we give the corresponding widths and heights as computed at different levels of theory. Cavity dimensions are defined as the interatomic distance between the farthest atoms of the cavity in a given direction. Cavity widths are comprised within 2.2 and $3.6 \mathrm{~nm}$ for the small cages, and within 3.1 and $4.4 \mathrm{~nm}$ for the large ones. Height-to-width ratios are typically in the range 0.5-0.6, except for PS ( 0.83) and TAL ( 0.37). Hence, we have a wide variety of cavities ranging from small pore for TAS to large pore for TPL. Inspection of this table reveals that, for a given monomeric compound, large cavities show, as expected, larger widths than small ones. In contrast, we compute similar heights for both types of cavities. Moreover, this table lists the dipole moments of these hosts, which are rather weak (around 0.1 debye), with the only exception of TS and TL, for which we calculate values of 1.0 and 1.2 debye, respectively. Tables 2, 3 and 4 give some chemical, energetic and spectroscopic parameters of these isolated cavities (cf. infra for more details).

The structures of DXR encapsulated in phenylene- (PS,PL), thiophene- (TS,TL), phenyl-cored thiophene- (TPS,TPL) and thioazole-based (TAS,TAL) dendrimer cavities optimized at the PBE+D3 / 6-31G (2df,p) level of theory are represented in Table 5. These structures correspond to the lowestenergy encapsulation complexes that we found after trying several initial geometric arrangements between the host and guest molecular units. We found that stable structures corresponding to partial encapsulation of DXR inside the cavities (not shown here) have binding energies of at least $10 \mathrm{kcal} \mathrm{mol}^{-}$ ${ }^{1}$ (in absolute values) smaller than the inclusion complexes. We note that the B3LYP+D3 / 6-31G (2df,p) approach leads to similar results as with PBE+D3 / 6-31G (2df,p).

While DRX does not present significant structural changes upon encapsulation, the structural modifications of the host cavities upon encapsulation are not only reflected by the substantial changes in the dihedral angles between atoms along the cavity backbone (cavity squeezing), but also by the nonnegligible changes in the X-H bond lengths of atom pairs of the cavity involved in hydrogen bonds with the guest molecule or simply lying close to it. For instance, Table 2 shows that the heights of the TAS and TAL cavities reduce from 15.7-17.6 $\AA$ to 8.0-10.7 $\AA$. This contraction favours the DRX-host 
interactions. Moreover, Table 5 displays, for the different complexes, a few selected interatomic distances for the closest interacting host-guest atom pairs and their Wiberg bond indices (WBIs), obtained using the PBE+D3 / 6-31G (2df,p) approach. The WBIs help in revealing the hydrogen bonds that may be crucial for the stability of the complexes and for their final structure. In particular, we remark the S-H13, S-H28 and $\mathrm{N}^{\prime}-\mathrm{H} 28$ bonds in the complexes involving the TS, TL and TAL cavities, respectively, with WBIs of 0.0272, 0.0317 and 0.0926, in the same order. Since these hydrogen bonds possess WBIs of about 0.03 or larger, they will play a major role in the encapsulation process of DXR in the above-mentioned cavities.

Even if it would be exceedingly difficult to predict the most favourable docking configurations of the guest molecule inside the cavity only on the basis of the electrostatic potential mappings, it can be verified a posteriori that the complexation geometries satisfy obvious electrostatic requirements of electron rich regions in the guest molecule approaching electron poor domains in the host cavity and vice versa. Figure 3 displays the optimized structures for the small and large dendrimer cages, with and without encapsulated doxorubicin, together with a representation of the electrostatic potential mapped over the ground-state isodensity surface. In the small cavities DXR is trapped at the centre of the host structure, the size of which is comparable to that of the guest molecule, thus maximizing the host-guest interactions. To further strengthen these interactions, a non-negligible cage squeezing occurs in the formation of DXR@TS and DXR@TAS complexes, where, at the PBE+D3 / 6-31G (2df,p) level, we calculate variations of cage heights of around $40 \%$ or more. Complexation in the bigger cavities results mainly in the DXR molecule trapped at cage corners, thus maximizing the stabilization induced by intermolecular forces in these larger systems. Again, as with small cavities, a remarkable cage squeezing occurs upon complexation in the case of DXR@TL and DXR@TAL.

In these complexes, apart from the van der Waals non-covalent interactions (e.g. $\pi$-stacking) additional stabilization may also come, at least in cavities containing electronegative atoms, from hydrogen bonding, so the lowest energy structures, in these cases, will result from the synergy between these two types of bonding (see, in particular, TS, TL and TAL complexes in Table 5). In sum, we have two dominant types of complexation: (i) H-bonding for PS, PL, TL, TPS, TPL, TAS or (ii) $\pi$-stacking for TS, TAL. This can be verified in most of the complexes displayed in Figure 3 by comparison with Figure 1b. Complexation tends to favour $\pi$ stacking interactions in the case of TS and TAL, mainly because of the non-zero dipole moment and the more positive $\mathrm{Q}_{z z}$ electric quadrupole component in thiophene and thiazole compared to benzene, while for the other complexes sigma type interactions are dominant. We note that $\pi$ stacking is correlated, with the only exception of TL, with a significant cage squeezing.

\section{b. Encapsulation energies of DXR in the cavities}


At all levels of theory adopted in this study, these complexes show similar stabilities, with encapsulation energies comprised within $\sim-34$ and $\sim-68 \mathrm{kcal} \mathrm{mol}^{-1}$. The effect of the applied theoretical method on the calculated energies strongly depends on the system. Small differences of 6.1 and $8.5 \mathrm{kcal}$ $\mathrm{mol}^{-1}$, between the maximum and minimum values obtained with the four approaches listed in Table S1 of the Supporting Information, are seen for DXR@TPS and DXR@PL, respectively, while larger differences are calculated for the other complexes, especially for DXR@TL (33.5 kcal mol $\left.{ }^{-1}\right)$ and DXR@TPL (21.9 kcal mol$\left.{ }^{-1}\right)$. These differences are imputable to both the chosen basis set and method. At the PBE+D3 / 6-31G (2df,p) level we found the following order of stability: DXR@TL(-68.21 kcal mol $\left.^{-1}\right)>$ DXR@TS(-66.86 kcal mol $\left.{ }^{-1}\right)>$ DXR@TAL(-62.16 kcal mol $\left.{ }^{-1}\right)>$ DXR@TAS(-45.14 kcal $\left.\mathrm{mol}^{-1}\right) \approx$ DXR@PS(-45.13 $\left.\mathrm{kcal} \mathrm{mol}^{-1}\right)>$ DXR@TPL(-36.02 kcal mol $\left.{ }^{-1}\right)>$ DXR@PL(-35.91 kcal $\left.\mathrm{mol}^{-1}\right)>$ DXR@TPS(-34.95 kcal mol $\left.{ }^{-1}\right)$. Empirical analysis based on chemical potentials, hardness and electrophilicity indices confirm this tendency. See Supporting Information for details.

Based solely on the energetics of the encapsulation process, all these systems can be employed as potential drug carriers. Indeed, the computed encapsulation energies of DRX fall in the ideal range for drug encapsulation, since the stabilization induced by complexation will be sufficient to confine DXR inside the dendrimers, while still allowing a relatively easy drug releasing into the blood stream, especially in the case of weakly polar cavities (i.e. those based on P and TP monomers in our case). In the host-guest complexes, the bonding interactions are expected to exhibit some covalent character, with a non-negligible electron transfer occurring between the host and guest entities. Therefore, encapsulation in this case will not be a purely physical process.

\section{c. Vibrational induced shifts upon encapsulation}

Dipole moments of the host structures are relatively low for the phenylene-, thiophene-, and phenyl-cored thiophene-based cages, where calculated values at the PBE+D3 / 6-31G (2df,p) level span the range of 0.0-0.72 debye. Due to the larger dipole moment of thiazole (1.61 \pm 0.03 debye[47]) compared to thiophene $(0.55 \pm 0.01$ debye [48]) TAS and TAL cages have larger dipole moments of 3.24 and 1.24 debye, respectively. Dipole moments of the complexed cages are larger, as a result of the rather large dipole moment of doxorubicin, which is calculated to be 6.68 debye at the PBE+D3 / 6-31G (2df,p) level (7.03 debye with B3LYP+D3 / 6-31G (2df,p).

The analysis of the IR spectra of DXR can be used to identify this molecular species in its free or complexed form. Additionally, intensity variations and shifts in the absorption peaks of the guest and host molecules can provide information on the structure and formation of these complexes. In Table 3 we give a selection of the most intense stretching and bending vibrational fundamentals for the isolated host cavities, guest molecule, and encapsulation complexes evaluated at the PBE+D3 / 6-31G (2df,p) level within the harmonic normal mode approximation. Inspection of these data shows that the strongest 
effects are seen in the $v(\mathrm{O} 5-\mathrm{H} 8)$ stretching frequency of DXR (H8 is an internally hydrogen-bonded proton), where red-shifts of roughly $20 \mathrm{~cm}^{-1}$ on average are calculated for the complexed forms, with largest deviations predicted for DXR@ PL $\left(-49 \mathrm{~cm}^{-1}\right)$. and DXR@ TL $\left(-41 \mathrm{~cm}^{-1}\right)$. As for the v(N1-H24) stretching, both positive and negative shifts are calculated: for the complexes involving PS, PL, TPL and TS cages we calculate blue-shifts of around $+8 \mathrm{~cm}^{-1}$ on average, while for TPS, TL, TAS, TAL redshifts of around $-10 \mathrm{~cm}^{-1}$ are found. Slightly red-shifted fundamentals are also calculated for the $v(\mathrm{C}-\mathrm{H})$ stretching and $\delta(\mathrm{C}=\mathrm{C})$ bending of DXR from aryl rings, with average absolute differences not exceeding $6 \mathrm{~cm}^{-1}$. The most intense transitions involving normal vibrations of atoms in the cavities do not display significant variations upon encapsulation, with the notable exceptions of the $v(\mathrm{C}-\mathrm{H})$ stretching from phenylene rings in PS $\left(+8 \mathrm{~cm}^{-1}\right)$, the $v(\mathrm{C}-\mathrm{H})$ stretching from thiazole units in TAL, and the $\delta(\mathrm{C}=\mathrm{C})$ bending of phenyl rings in TPS. Therefore, the O5-H8 stretching can be used as specific probe of DXR encapsulation.

\section{d. Electronic transitions}

Figures 4 and 5 show HOMO and LUMO molecular orbitals (MOs) with the corresponding energy gaps for isolated and complexed DXR, involving small and large cavities, obtained at the PBE/6$31 \mathrm{G}(2 \mathrm{df}, \mathrm{p})$ level. The fact that in all complexes, apart from DXR@TAS, HOMO and LUMO orbitals are not localized on the same molecular entity (either guest or host), suggests that the encapsulation process will modify the electronic structure of the DXR molecule to an appreciable extent. This further emphasizes the expected partial covalent character of the binding interaction within encapsulated DXR. We note that the HOMO is localised in most cases on the dendrimer cage, while the LUMO lies on the DXR unit. The HOMO-LUMO gaps are comprised within 0.43 and $1.30 \mathrm{eV}$, so the corresponding transitions fall in the near IR spectrum and should be accompanied by significant charge transfer, with the cage acting as an electron donor and the DXR as an acceptor.

The comparison of the calculated frontier MO energy gaps indicates that complexed DXR will be more chemically active than free DXR. In particular, large negative energy gap variations are calculated for TPS $(-0.871 \mathrm{eV})$, TPL $(-0.626 \mathrm{eV})$ and TL $(-0.626 \mathrm{eV})$. Similarly, energy gaps are smaller in the complexed cages compared to the free host structures. This is particularly the case for PS and PL, for which the energy gaps get smaller by -1.714 and $-1.686 \mathrm{eV}$, respectively.

As for the IR spectra, the formation of supramolecular assemblies can be effectively monitored by inspecting the variations of the absorption peaks in the inherent UV-Vis spectra induced by complexation. In the present study we used the TD-DFT approach to identify the most intense lowenergy electronic transitions that could be used to distinguish complexed from free DXR. The results of this study are summarized in Table 4, where the wavelength maxima of the most intense electronic bands of DXR, host cavities and complexes are reported together with the corresponding oscillator strengths. These data are obtained with the B3LYP functional and the 6-31G(2df,p) basis of atomic functions, 
because this methodology leads to the most accurate predictions of transition peaks and relative intensities for the UV-Vis spectrum of isolated DXR.

Strong absorption wavelengths for isolated DXR are calculated at 476, 298 and $264 \mathrm{~nm}$. The experimental spectrum of DXR presents two intense bands, one at around $480 \mathrm{~nm}$ and another one at $290 \mathrm{~nm}$ [49], that is in excellent agreement with the calculated values. These bands corresponds to the $\mathrm{HOMO} \rightarrow \mathrm{LUMO}\left(\mathrm{S}_{0} \rightarrow \mathrm{S}_{1}\right.$ at $\left.476 \mathrm{~nm}\right), \mathrm{HOMO} \rightarrow \mathrm{LUMO}+2\left(\mathrm{~S}_{0} \rightarrow \mathrm{S}_{3}\right.$ at $\left.298 \mathrm{~nm}\right)$ and HOMO$2 \rightarrow$ LUMO+1 $\left(\mathrm{S}_{0} \rightarrow \mathrm{S}_{4}\right.$ at $\left.264 \mathrm{~nm}\right)$ transitions.

Strong absorption bands are calculated for all the considered cages, where, respectively, PS / PL have maximum absorption peaks at 316 / $365 \mathrm{~nm}$, TPS / TPL at 580 / $618 \mathrm{~nm}$, TS / TL at 594 / 596 $\mathrm{nm}$, and TAS / TAL at $556 / 570 \mathrm{~nm}$. We note that, for a given dendrimer type, the absorption maximum in the large cage is generally red-shifted with respect to the small one, as one would expect from delocalization of the molecular orbitals involved in the transition over a larger region. Bare cage spectra present intense bands in the UV-Vis region that are quenched after complexation with DXR, notably in the case of DX@PS where the reduction in the total absorption intensity is 75\%. From the comparison of the absorption maxima of complexed DXR with respect to bare cages, we note that appreciable red shifts are calculated for PS and TPS, while blue shifts are predicted for TPL, TS, TL and TAL.

\section{Conclusions}

Using first principle methodologies, we showed that encapsulation of DXR inside dendritic structures from polymerization of phenylene-, thiophene-, phenyl-cored thiophen- and thioazolemonomeric units is feasible. These systems are good candidates to be employed as drug carriers in biological media. Encapsulation in these molecular cages occurs by various types of weak interactions including van der Waals, electrostatic and hydrogen bonding, where the most stable encapsulation geometries include a variety of $\pi-\pi$ stacking configurations and $\sigma$-type interactions. Specifically, we show that encapsulation is governed by both $\pi-\pi$ stacking and $\sigma$-type interactions, with important contributions, for some of the considered structures, from hydrogen bonding as well. Encapsulation energies are comprised within around -30 and $-70 \mathrm{kcal} \mathrm{mol}^{-1}$, that is in the ideal range for reversible encapsulation. The cages considered in this study are highly flexible, and can be used to arrange several types of substituted DXR, or similar molecular guests. Improvements of the present models can be provided by full account of the dendritic structures, and by introducing explicit/implicit simulations of the water solvent. However, explicit solvent simulations with the full dendrimer complexes, given the large size of the inherent molecular frameworks, would be computationally very demanding. In addition, we showed that shifts of the O5-H8 stretching frequency of DXR, and UV-Vis spectroscopy can be used as specific probes of encapsulation-induced effects. 


\section{References}

[1] X. Ma and Y. Zhao, Chem. Rev. 115, 7794-7839 (2015).

[2] K. Ariga and T. Kunitake, Supramolecular Chemistry - Fundamentals and Applications: Advanced Textbook, Springer Berlin Heidelberg, 2006.

[3] A. Yousaf, S. A. Hamid, N. M. Bunnori, and A. A. Ishola, Drug Des. Devel. Ther 9, 2831-2838 (2015).

[4] E. Buhleier, W. Wehner, and F. Vögtle, Synthesis 1978, 155-158 (1978).

[5] D. A. Tomalia, J. R. Dewald, M. R. Hall, S. J. Martin, and P. B. Smith, Preprints of the $1^{\text {st }}$ SPSJ Int. Polym. Conf., Soc. Polym. Sci. Jpn., Kyoto, 1984, 65.

[6] D. A. Tomalia, H. Baker, J. Dewald, M. Hall, G. Kallos, S. Martin, J. Roeck, J. Ryder and P. Smith, Polymer Journal 17, 117-132 (1985).

[7] P. G. de Gennes and H. Hervet, J. Phys. Lett. (Paris) 44 , L351-L360 (1983).

[8] J. F. Jansen, E. M. de Brabander-van den Berg, and E. W. Meijer, Science 266, 1226-1229 (1994).

[9] P. K. Maiti, T. Çağin, S.-T. Lin, and W. A. Goddard, III, Macromolecules 38, 979-991 (2005).

[10] R. Brodbeck, T. Tönsing, D. Andrae, and D. Volkmer, J. Phys. Chem. B 112, 5153-5162 (2008).

[11] A. Rahimi, S. Amjad-Iranagh, and H. Modarress, J. Mol. Model. 22:59 (2016).

[12] P.-Y. Yang, S.-P. Ju, Y.-C. Chuang, and H.-Y. Chen, Comput. Mater. Sci. 137, 144-152 (2017).

[13] O. Tacar, P. Sriamornsak, and C. R. Dass, J. Pharm. Pharmacol. 65, 157-170 (2013).

[14] G. Aubel-Sadron, and D. Londos-Gagliardi, Biochimie 66, 333-352 (1984).

[15] W. J. Pigram, W. Fuller, and L. D. Hamilton, Nature New Bio. 235, 17-19 (1972).

[16] M. Frisch et al. GAUSSIAN 09, Revision D.01, Gaussian, Inc., Wallingford CT, 2013.

[17] J. P. Perdew, K. Burke, and M. Ernzerhof, Phys. Rev. Lett. 77, 3865-3868 (1996).

[18] A. D. Becke, J. Chem. Phys. 98, 5648-5652 (1993).

[19] C. T. Lee, W. T. Yang, and R. G. Parr., Phys. Rev. B 37, 785-789 (1988).

[20] W. J. Hehre, R. Ditchfield, and J. A. Pople, J. Chem. Phys. 56, 2257-2261 (1972).

[21] M. M. Francl, W. J. Pietro, W. J. Hehre, J. S. Binkley, M. S. Gordon, D. J. DeFrees, and J. A. Pople, J. Chem. Phys. 77, 3654-3665 (1982).

[22] L. A. Curtiss, K. Raghavachari, P. C. Redfern, V. Rassolov, and J. A. Pople, J. Chem. Phys. 109, 7764-7776 (1998).

[23] S. Grimme, J. Antony, S. Ehrlich, and H. Krieg, J. Chem. Phys. 132, 154104-19 (2010).

[24] R. Sedlak, T. Janowski, M. Pitoňák, J. Řezáč, P. Pulay, and P. Hobza, J. Chem. Theory Comput. 9, 3364-3374 (2013).

[25] J. Tao, J. P. Perdew, V. N. Staroverov, and G. E. Scuseria, Phys. Rev. Lett. 91, 146401-04 (2003).

[26] N. Marom, A. Tkatchenko, M. Rossi, V. V. Gobre, O. Hod, M. Scheffler, and L. Kronik, J. Chem. Theory Comput. 7, 3944-3951 (2011). 
[27] Y. Zhao and D. G. Truhlar, J. Chem. Theory Comput. 3, 289-300 (2007).

[28] S. Tsuzuki and H. P. Lüthi, J. Chem. Phys. 114, 3949-3957 (2001).

[29] G. A. DiLabio, E. R. Johnson, and A. Otero-de-la-Roza, Phys. Chem. Chem. Phys. 15, 1282112828 (2013).

[30] J. Wang, R. M. Wolf, J. W. Caldwell, P. A. Kollman, and D. A. Case, J. Comput. Chem. 25, 1157-1174 (2004).

[31] J. P. Foster and F. Weinhold, J. Am. Chem. Soc. 102, 7211-7218 (1980).

[32] E. Runge and E. K. U. Gross, Phys. Rev. Lett. 52, 997-1000 (1984).

[33] R. Bauernschmitt and R. Ahlrichs, Chem. Phys. Lett. 256, 454-464 (1996).

[34] M. E. Casida, C. Jamorski, K. C. Casida, and D. R. Salahub, J. Chem. Phys. 108, 4439-4449 (1998).

[35] R. E. Stratmann, G. E. Scuseria, and M. J. Frisch, J. Chem. Phys. 109, 8218-8224 (1998).

[36] F. Furche and R. Ahlrichs, J. Chem. Phys. 117, 7433-7447 (2002).

[37] D. Jacquemin, B. Mennucci, and C. Adamo, Phys. Chem. Chem. Phys. 13, 16987-16998 (2011).

[38] C. Adamo and D. Jacquemin, Chem. Soc. Rev. 42, 845-856 (2013).

[39] B. C. M. A. Ashwin, A. H. A. Baby, M. Prakash, M. Hochlaf, and P. M. Mareeswaran, J. Phys. Org. Chem. 31:e3788 (2017).

[40] M. Prakash, K. Mathivon, D. M. Benoit, G. Chambaud, and M. Hochlaf, Phys. Chem. Chem. Phys. $16,12503-12509$ (2014).

[41] S. Kamalakannan, M. Prakash, G. Chambaud, and M. Hochlaf, ACS Omega 3, 18039-18051 (2018).

[42] H. C. Gottschalk, A. Poblotzki, M. A. Suhm, M. M. Al-Mogren, J. Antony, A. A. Auer, L. Baptista, D. M. Benoit, G. Bistoni, F. Bohle, R. Dahmani, D. Firaha, S. Grimme, A. Hansen, M. E. Harding, M. Hochlaf, C. Holzer, G. Jansen, W. Klopper, W. A. Kopp, L. C. Kröger, K. Leonhard, H. Mouhib, F. Neese, M. N. Pereira, I. S. Ulusoy, A. Wuttke, and R. A. Mata, J. Chem. Phys. 148, 014301-13 (2018). [43] R. Boulmène, K. Boussouf, M. Prakash, N. Komiha, M. M. Al-Mogren, and M. Hochlaf, ChemPhysChem 17, 994-1005 (2016).

[44] R. Boulmène, M. Prakash, and M. Hochlaf, Phys. Chem. Chem. Phys. 18, 29709-29720 (2016).

[45] N. Pandey and K. Mahara, PharmaTutor 5, 48-54 (2017).

[46] S. Tripathy and M. K. Das, J. Appl. Pharm. Sci. 3, 142-149 (2013).

[47] B. Bak, D. Christensen, L. Hansen-Nygaard, and J. Rastrup-Andersen, J. Mol. Spectrosc. 9, 222224 (1962).

[48] T. Ogata and K. Kozima, J. Mol. Spectrosc. 42, 38-46 (1972).

[49] N. S. H. Motlagh, P. Parvin, F. Ghasemi, and F. Atyabi, Biomed. Opt. Express 7, 2400-2406 (2016).

[50] M. L. Laury, M. J. Carlson, and A. K. Wilson, J. Comput. Chem. 33, 2380-2387 (2012). 
Table 1: Selected optimized bond lengths $(\AA)$ and dihedral angles (degrees) for isolated doxorubicin cal culated using the B3LYP+D3 and PBE+D3 DFTs. For atom numbering see Figure1.

\begin{tabular}{|c|c|c|c|c|}
\hline & \multicolumn{2}{|c|}{ B3LYP+D3 } & \multicolumn{2}{|c|}{$\mathrm{PBE}+\mathrm{D} 3$} \\
\hline Bond/Angle & $6-31 \mathrm{G}$ & 6-31G (2df,p) & $6-31 \mathrm{G}$ & 6-31G (2df,p) \\
\hline $\mathrm{C} 14-\mathrm{H} 3$ & 1.082 & 1.081 & 1.091 & 1.090 \\
\hline $\mathrm{C} 11-\mathrm{O} 1$ & 1.376 & 1.345 & 1.382 & 1.351 \\
\hline $\mathrm{C} 5-\mathrm{O} 4$ & 1.367 & 1.339 & 1.366 & 1.336 \\
\hline $\mathrm{C} 2-\mathrm{O} 5$ & 1.368 & 1.344 & 1.372 & 1.346 \\
\hline $\mathrm{C} 19-\mathrm{O} 7$ & 1.457 & 1.421 & 1.464 & 1.426 \\
\hline $\mathrm{C} 17-\mathrm{O} 6$ & 1.475 & 1.437 & 1.488 & 1.448 \\
\hline $\mathrm{C} 21-\mathrm{O} 9$ & 1.446 & 1.407 & 1.455 & 1.415 \\
\hline $\mathrm{C} 24-\mathrm{N} 1$ & 1.467 & 1.465 & 1.470 & 1.469 \\
\hline $\mathrm{O} 4-\mathrm{H} 7$ & 1.006 & 0.993 & 1.041 & 1.028 \\
\hline $\mathrm{O} 5-\mathrm{H} 8$ & 0.991 & 0.974 & 1.010 & 0.990 \\
\hline $\mathrm{O} 10-\mathrm{H} 23$ & 0.985 & 0.968 & 1.002 & 0.980 \\
\hline $\mathrm{C} 8=\mathrm{O} 2$ & 1.276 & 1.241 & 1.296 & 1.261 \\
\hline $\mathrm{C} 7=\mathrm{O} 3$ & 1.247 & 1.215 & 1.262 & 1.229 \\
\hline $\mathrm{C} 20=\mathrm{O} 8$ & 1.246 & 1.214 & 1.258 & 1.226 \\
\hline $\mathrm{C} 19-\mathrm{C} 20$ & 1.522 & 1.550 & 1.522 & 1.528 \\
\hline H5-C15-O1-C11 & -179.79 & -179.08 & -179.56 & -179.08 \\
\hline H7-O4-C5-C6 & -179.61 & -179.52 & -179.92 & -179.52 \\
\hline H8-O5-C2-C3 & -176.42 & -174.52 & -175.20 & -174.52 \\
\hline $\mathrm{C} 21-\mathrm{O} 6-\mathrm{C} 17-\mathrm{C} 1$ & 140.77 & 131.22 & 137.81 & 131.22 \\
\hline $\mathrm{H} 23-\mathrm{O} 10-\mathrm{C} 25-\mathrm{C} 24$ & -161.91 & -160.96 & -158.96 & -158.74 \\
\hline $\mathrm{H} 24-\mathrm{N} 1-\mathrm{C} 24-\mathrm{C} 25$ & -153.19 & -166.69 & -154.41 & -166.69 \\
\hline $\mathrm{H} 28-\mathrm{O} 11-\mathrm{C} 21-\mathrm{C} 20$ & 0.177 & -1.35 & -0.33 & -1.35 \\
\hline
\end{tabular}


Table 2: PBE+D3 / 6-31G (2df,p) cavity widths (W, ̊) and heights (H, ̊̊) with and without DRX. For DRX encapsulated species, we give also the dipole moments ( $\mu$, debye) and the encapsulation energies (E, kcal mol ${ }^{-1}$ ) of doxorubicin trapped in PS, PL, TS, TL, TPS, TPL, TAS and TAL cavities. Table S1 lists the corresponding values as computed at the B3LYP+D3 / 6-31G, B3LYP+D3 / 6-31G (2df,p) and PBE+D3 / 6-31G levels of theory.

\begin{tabular}{|c|c|c|c|c|}
\hline Cavity type & $\mathrm{W}$ & $\mathrm{H}$ & $\mu$ & $\mathrm{E}$ \\
\hline $\mathrm{PS}$ & 22.34 & 18.64 & 0.13 & \\
\hline $\mathrm{PL}$ & 30.42 & 19.92 & 0.25 & \\
\hline TS & 35.66 & 18.94 & 0.72 & \\
\hline $\mathrm{TL}$ & 36.34 & 23.48 & 0.57 & \\
\hline TPS & 33.87 & 21.19 & 0.16 & \\
\hline TPL & 40.84 & 22.23 & 0.00 & \\
\hline TAS & 33.51 & 17.63 & 3.24 & \\
\hline TAL & 41.73 & 15.70 & 1.24 & \\
\hline DXR@PS & 22.52 & 16.86 & 6.57 & -45.13 \\
\hline DXR@PL & 32.50 & 16.86 & 5.78 & -35.91 \\
\hline DXR@TS & 37.52 & 10.25 & 4.18 & -66.86 \\
\hline DXR@TL & 35.78 & 13.45 & 5.70 & -68.21 \\
\hline DXR@TPS & 34.24 & 21.52 & 4.77 & -34.95 \\
\hline DXR@TPL & 40.94 & 21.56 & 6.37 & -36.02 \\
\hline DXR@TAS & 33.67 & 10.74 & 7.81 & -45.14 \\
\hline DXR@TAL & 45.15 & 7.96 & 4.46 & -62.16 \\
\hline
\end{tabular}


Table 3: Some selected PBE+D3 / 6-31G (2df,p) harmonic vibrational frequencies in $\mathrm{cm}^{-1}(v=$ stretching, $\delta=$ bending) of isolated hosts, DRX and host-guest complexes. Index ph is for aryl rings of DXR, phy for phenylen rings, thio for thiophen rings and thiaz for thioazol rings of the host cavities. Anharmonic frequencies can be deduced from harmonic ones after applying the scaling factors defined in Ref. [50].

\begin{tabular}{|c|c|c|c|c|c|c|c|c|c|c|}
\hline Compound & $v\left(\mathrm{O}_{5}-\mathrm{H}_{8}\right)$ & $v\left(\mathrm{~N}_{1}-\mathrm{H}_{24}\right)$ & $v(\mathrm{C}-\mathrm{H})^{p h}$ & $v(\mathrm{C}-\mathrm{H})^{p h y}$ & $v(\mathrm{C}-\mathrm{H})^{\text {thio }}$ & $v(\mathrm{C}-\mathrm{H})^{\text {thiaz }}$ & $\delta(\mathrm{C}=\mathrm{C})^{p h}$ & $\delta(\mathrm{C}=\mathrm{C})^{\text {phy }}$ & $\delta(\mathrm{C}=\mathrm{C})^{\text {thio }}$ & $\delta(\mathrm{C}=\mathrm{C})^{\text {thiaz }}$ \\
\hline DXR & 3119 & 3565 & 3175 & & & & 1430 & & & \\
\hline PS & & & & 3118 & & & & 1492 & & \\
\hline PL & & & & 3129 & & & & 1492 & & \\
\hline TPS & & & & 3132 & 3163 & & & 1500 & 1493 & \\
\hline TPL & & & & 3132 & 3161 & & & 1515 & 1495 & \\
\hline TS & & & & & 3163 & & & & 1506 & \\
\hline TL & & & & & 3163 & & & & 1513 & \\
\hline TAS & & & & & & 3193 & & & & 1445 \\
\hline TAL & & & & & & 3191 & & & & 1447 \\
\hline DXR@PS & 3105 & 3579 & 3169 & 3126 & & & 1426 & 1493 & & \\
\hline DXR@PL & 3070 & 3576 & 3170 & 3129 & & & 1430 & 1493 & & \\
\hline DXR@TPS & 3108 & 3563 & 3170 & 3131 & 3163 & & 1425 & 1512 & 1492 & \\
\hline DXR@TPL & 3112 & 3572 & 3175 & 3133 & 3162 & & 1429 & 1512 & 1494 & \\
\hline DXR@TS & 3125 & 3566 & 3179 & & 3160 & & 1426 & & 1510 & \\
\hline DXR@TL & 3078 & 3538 & 3171 & & 3161 & & 1429 & & 1513 & \\
\hline DXR@TAS & 3113 & 3554 & 3174 & & & 3191 & 1427 & & & 1447 \\
\hline DXR@TAL & 3092 & 3564 & 3178 & & & 3199 & 1427 & & & 1441 \\
\hline
\end{tabular}


Table 4: Wavelength maxima $\left(\lambda_{\max }, \mathrm{nm}\right)$ and oscillator strengths (f) of bands in the electronic spectra of the cavities, DRX and their complexes obtained at the TD-DFT B3LYP/6-31G level of theory.

\begin{tabular}{|c|c|c|c|c|c|c|c|c|c|c|c|c|c|c|c|c|c|c|}
\hline \multicolumn{2}{|l|}{ Transition } & DXR & PS & DXR@PS & PL & DXR@PL & TPS & DXR@TPS & TPL & DXR@TPL & TS & DXR@TS & $\mathrm{TL}$ & DXR@TL & TAS & DXR@TAS & TAL & DXR@TAL \\
\hline \multirow{2}{*}{$\mathrm{HOMO} \rightarrow \mathrm{LUMO}$} & $\lambda$ & 476 & & & & & 580 & & & & & & & & & 622 & & \\
\hline & f & 0.22 & & & & & 4.26 & & & & & & & & & 0.11 & & \\
\hline \multirow{2}{*}{ HOMO-1 $\rightarrow$ LUMO } & $\lambda$ & & & & 365 & & & & & & & & & & 556 & 555 & 570 & 615 \\
\hline & $\mathrm{f}$ & & & & 5.72 & & & & & & & & & & 2.63 & 0.84 & 5.66 & 0.29 \\
\hline \multirow{2}{*}{ HOMO-2 $\rightarrow$ LUMO } & $\lambda$ & & & & 325 & & & & & & & 576 & & & 507 & & 503 & \\
\hline & $\mathrm{f}$ & & & & 0.76 & & & & & & & 0.40 & & & 0.43 & & 0.19 & \\
\hline \multirow{2}{*}{ HOMO-3 $\rightarrow$ LUMO } & $\lambda$ & & 287 & & & & 475 & 477 & 518 & & 476 & & 505 & 502 & & & & 555 \\
\hline & $\bar{f}$ & & 0.14 & & & & 0.30 & 0.15 & 0.14 & & 0.49 & & 0.45 & 1.09 & & & & 2.41 \\
\hline \multirow{2}{*}{ HOMO-4 $\rightarrow$ LUMO } & $\lambda$ & & & 479 & & 477 & & & & & & 493 & & 491 & & & & \\
\hline & $\mathrm{f}$ & & & 0.19 & & 0.19 & & & & & & 0.25 & & 0.59 & & & & \\
\hline \multirow{2}{*}{$\mathrm{HOMO} \rightarrow \mathrm{LUMO}+1$} & $\lambda$ & & 316 & & & & & & 618 & 640 & 594 & 625 & 596 & & & 551 & & 579 \\
\hline & $\mathrm{f}$ & & 3.71 & & & & & & 5.64 & 1.23 & 4.10 & 0.41 & 2.53 & & & 1.67 & & 1.23 \\
\hline \multirow{2}{*}{$\mathrm{HOMO} \rightarrow \mathrm{LUMO}+2$} & $\lambda$ & 298 & 286 & & 323 & 376 & & & & & & & & & 512 & 546 & 515 & 553 \\
\hline & $\mathrm{f}$ & 0.05 & 0.27 & & 0.17 & 1.08 & & & & & & & & & 0.51 & 0.46 & 0.47 & 0.72 \\
\hline \multirow{2}{*}{$\mathrm{HOMO} \rightarrow \mathrm{LUMO}+3$} & $\lambda$ & & & & & & & & & 518 & & & & & & & & \\
\hline & $\mathrm{f}$ & & & & & & & & & 1.56 & & & & & & & & \\
\hline \multirow{2}{*}{ HOMO- $1 \rightarrow$ LUMO+1 } & $\bar{\lambda}$ & & & & & & 566 & 602 & & & 588 & & & 586 & 537 & & & \\
\hline & $\mathrm{f}$ & & & & & & 0.22 & 4.05 & & & 0.67 & & & 0.36 & 0.79 & & & \\
\hline \multirow{2}{*}{ HOMO- $1 \rightarrow$ LUMO+2 } & $\lambda$ & & & 337 & & & 483 & & & 587 & 557 & 560 & 543 & & & & & \\
\hline & $\mathrm{f}$ & & & 1.05 & & & 1.53 & & & 3.77 & 0.75 & 3.06 & 1.82 & & & & & \\
\hline \multirow{2}{*}{ HOMO- $1 \rightarrow$ LUMO+3 } & $\lambda$ & & & & & & & 502 & & & & & & & & & 511 & \\
\hline & $\mathrm{f}$ & & & & & & & 0.92 & & & & & & & & & 0.15 & \\
\hline \multirow{2}{*}{ HOMO-2 $\rightarrow$ LUMO+1 } & $\bar{\lambda}$ & 264 & & & & 367 & & & 522 & & & & 538 & 518 & & & & \\
\hline & $\mathrm{f}$ & 0.06 & & & & 4.31 & & & 1.78 & & & & 1.28 & 0.25 & & & & \\
\hline
\end{tabular}




\begin{tabular}{|c|c|c|c|c|c|c|c|c|c|c|c|c|c|}
\hline \multirow{2}{*}{$\mathrm{HOMO}-2 \rightarrow \mathrm{LUMO}+2$} & $\lambda$ & 282 & & 428 & & 465 & & 512 & 491 & 522 & 488 & & \\
\hline & $\mathrm{f}$ & 1.71 & & 0.43 & & 0.81 & & 0.24 & 0.54 & 2.02 & 0.34 & & \\
\hline \multirow{2}{*}{ HOMO- $2 \rightarrow$ LUMO +3} & $\lambda$ & & 295 & & 447 & 459 & 436 & 448 & 488 & 489 & & 454 & 473 \\
\hline & $\mathrm{f}$ & & 1.30 & & 0.22 & 0.16 & 0.33 & 0.32 & 0.31 & 0.20 & & 1.23 & 0.41 \\
\hline
\end{tabular}


Table 5: DRX encapsulated in PS, PL, TS, TL, TPS, TPL, TAS and TAL cavities. Structures are optimized at the PBE+D3/6-31G (2df,p) level of theory. We give also the main XH (X=N,O,S) guesthost bond lengths $(\AA)$. For the cavity type designation, see text. The images have been zoomed in to highlight the guest-host interactions.

\begin{tabular}{|c|c|c|c|c|}
\hline Cavity type & DXR@dendrimer cavities complex & Bond & Bond length & $\begin{array}{c}\text { Wiberg } \\
\text { bond } \\
\text { index }\end{array}$ \\
\hline PS & & $\begin{array}{l}\mathrm{O}_{4}-\mathrm{H}_{\mathrm{a}} \\
\mathrm{O}_{7}-\mathrm{H}_{\mathrm{a}} \\
\mathrm{O}_{10}-\mathrm{H}_{\mathrm{a}} \\
\mathrm{O}_{11}-\mathrm{H}_{\mathrm{a}}\end{array}$ & $\begin{array}{l}2.79 \\
2.60 \\
2.62 \\
2.98\end{array}$ & $\begin{array}{l}0.0021 \\
0.0515 \\
0.0063 \\
0.0013\end{array}$ \\
\hline PL & & $\begin{array}{l}\mathrm{O}_{1}-\mathrm{H}_{\mathrm{a}} \\
\mathrm{O}_{3}-\mathrm{H}_{\mathrm{a}} \\
\mathrm{O}_{4}-\mathrm{H}_{\mathrm{a}} \\
\mathrm{O}_{5}-\mathrm{H}_{\mathrm{a}} \\
\mathrm{O}_{7}-\mathrm{H}_{\mathrm{a}} \\
\mathrm{O}_{10}-\mathrm{H}_{\mathrm{a}} \\
\mathrm{O}_{11}-\mathrm{H}_{\mathrm{a}}\end{array}$ & $\begin{array}{l}2.61 \\
2.66 \\
2.72 \\
3.02 \\
2.64 \\
3.05 \\
2.60\end{array}$ & $\begin{array}{l}0.0050 \\
0.0028 \\
0.0034 \\
0.0037 \\
0.0095 \\
0.0014 \\
0.0117\end{array}$ \\
\hline $\mathrm{TS}$ & & $\begin{array}{l}\mathrm{O}_{2}-\mathrm{Hb} \\
\mathrm{O}_{5}-\mathrm{H}_{\mathrm{b}} \\
\mathrm{O}_{8}-\mathrm{H}_{\mathrm{b}} \\
\mathrm{O}_{10}-\mathrm{H}_{\mathrm{b}} \\
\mathrm{S}-\mathrm{H}_{13}\end{array}$ & $\begin{array}{c}2.83 \\
2.84 \\
2.81 \\
2.58 \\
2.80\end{array}$ & $\begin{array}{l}0.0030 \\
0.0034 \\
0.0027 \\
0.0139 \\
0.0272\end{array}$ \\
\hline TL & & $\begin{array}{l}\mathrm{O}_{2}-\mathrm{H}_{\mathrm{b}} \\
\mathrm{O}_{3}-\mathrm{H}_{\mathrm{b}} \\
\mathrm{O}_{5}-\mathrm{H}_{\mathrm{b}} \\
\mathrm{O}_{7}-\mathrm{H}_{\mathrm{b}} \\
\mathrm{O}_{11}-\mathrm{H}_{\mathrm{b}} \\
\mathrm{S}-\mathrm{H}_{28}\end{array}$ & $\begin{array}{l}2.91 \\
2.30 \\
2.54 \\
2.30 \\
2.39 \\
2.66\end{array}$ & $\begin{array}{l}0.0020 \\
0.0239 \\
0.0029 \\
0.0216 \\
0.0165 \\
0.0317\end{array}$ \\
\hline TPS & & $\begin{array}{l}\mathrm{O}_{2}-\mathrm{H}_{\mathrm{c}} \\
\mathrm{O}_{3}-\mathrm{H}_{\mathrm{c}} \\
\mathrm{O}_{4}-\mathrm{H}_{\mathrm{c}} \\
\mathrm{O}_{7}-\mathrm{H}_{\mathrm{c}} \\
\mathrm{O}_{10}-\mathrm{H}_{\mathrm{c}} \\
\mathrm{O}_{11}-\mathrm{H}_{\mathrm{c}}\end{array}$ & $\begin{array}{l}2.45 \\
2.51 \\
2.36 \\
2.58 \\
2.76 \\
2.58\end{array}$ & $\begin{array}{l}0.0050 \\
0.0045 \\
0.0127 \\
0.0056 \\
0.0057 \\
0.0089\end{array}$ \\
\hline
\end{tabular}




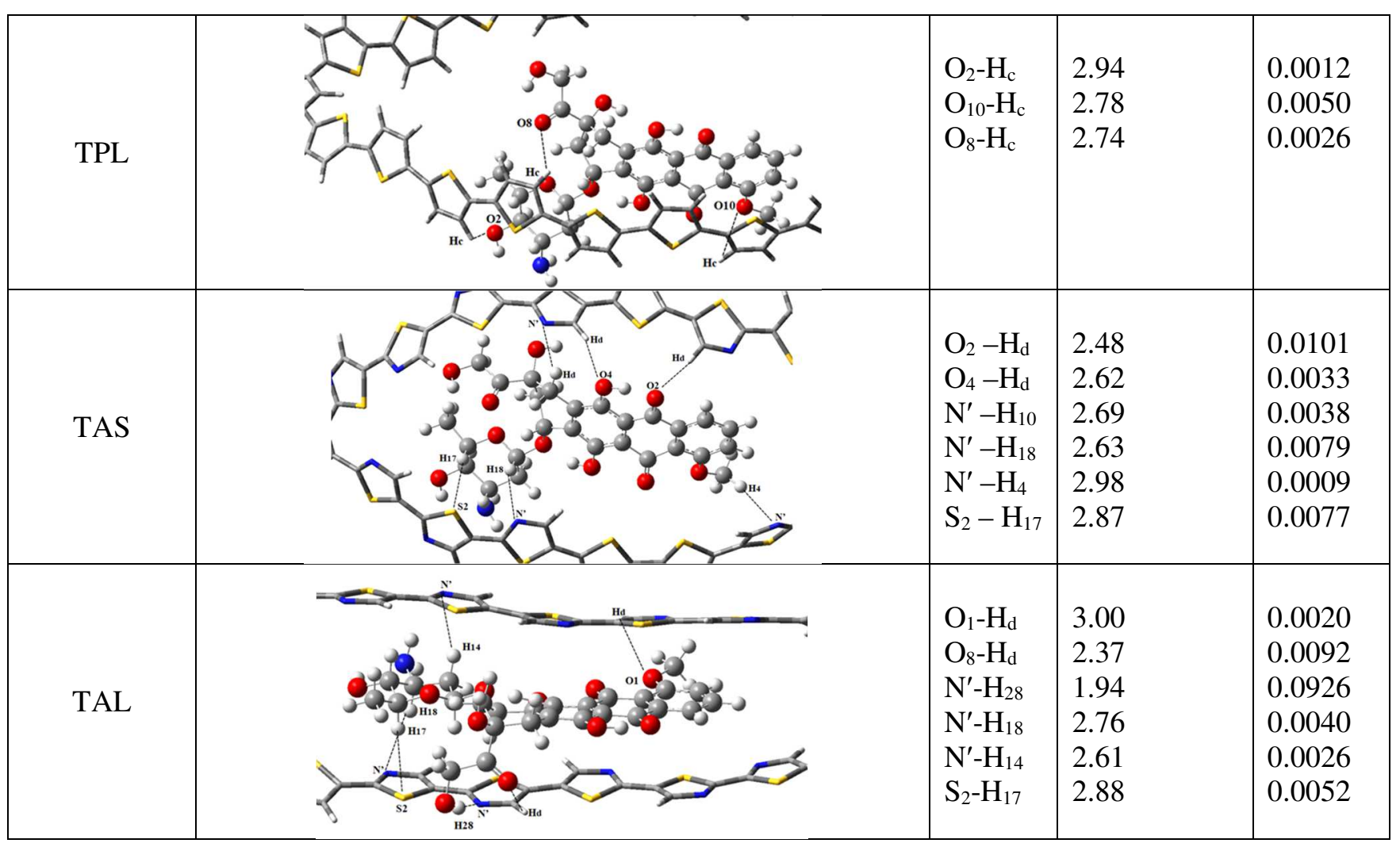


a)

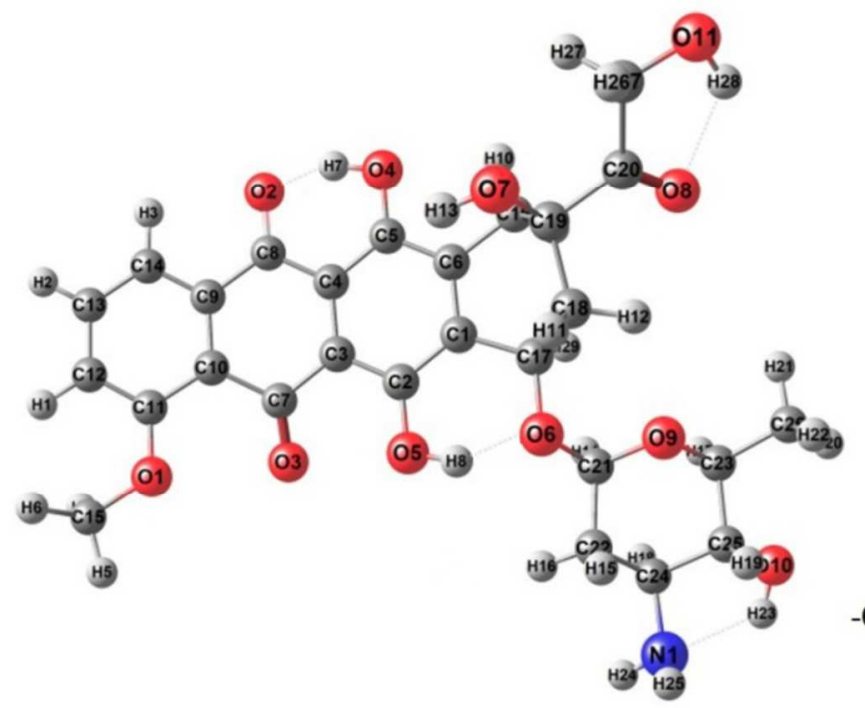

b)

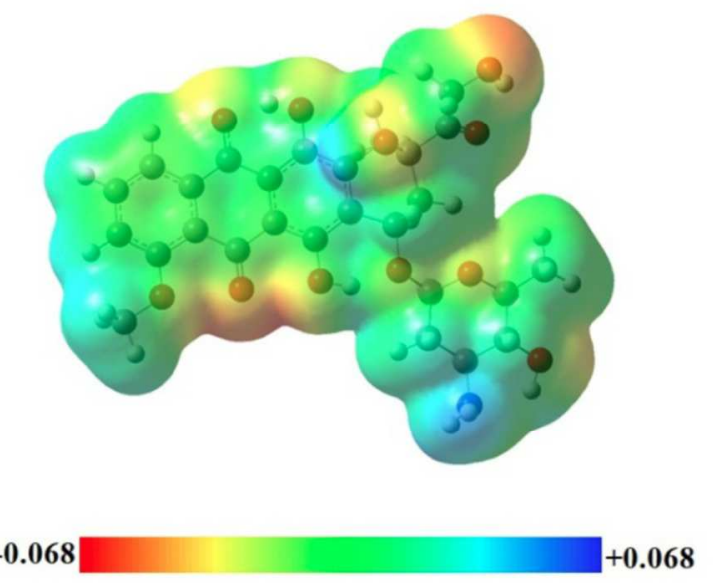

Figure 1: a) PBE+D3 / 6-31 G(2df,p) optimized equilibrium geometry of DRX. The C, O, H, and N atoms are grey, red, white, and blue, respectively. b) 3D molecular electrostatic potential (3D MEP) of DRX (from -0.068 to 0.068 a.u.) mapped over the ground-state electronic isodensity surface (set at 0.02 a.u.). 


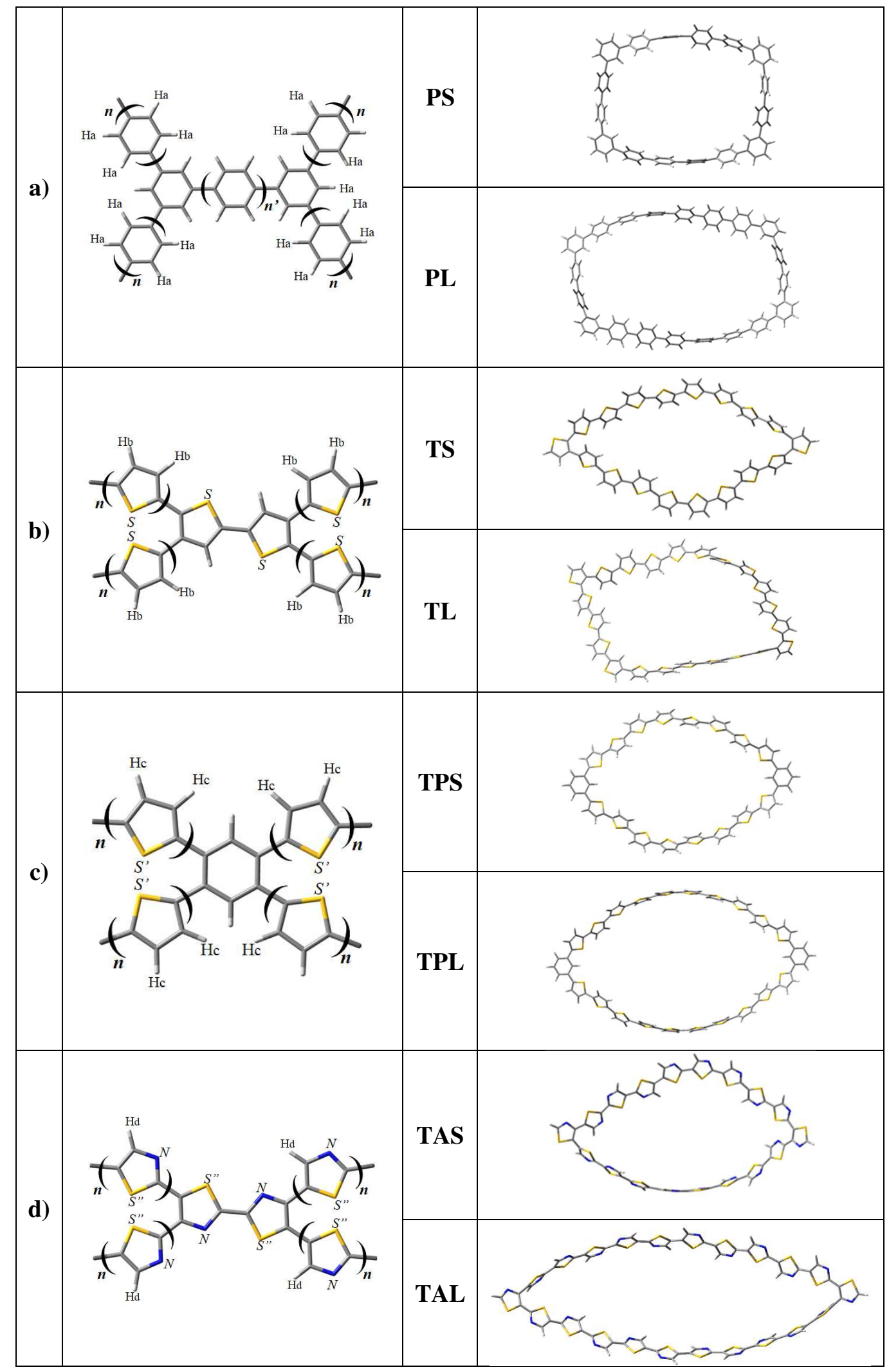

Figure 2: PBE+D3 / 6-31 G(2df,p) equilibrium optimized structures of a) phenylene- (P), b) thiophene$(\mathrm{T}), \mathrm{c})$ phenyl-cored thiophene- (TP) and d) thioazole-based (TA) monomers, and related small $(\mathrm{n}=3)$ and large $(\mathrm{n}=5)$ dendrimer cavities. The $\mathrm{C}, \mathrm{O}, \mathrm{H}$, and $\mathrm{N}$ atoms are displayed in grey, red, white, and blue, respectively. 

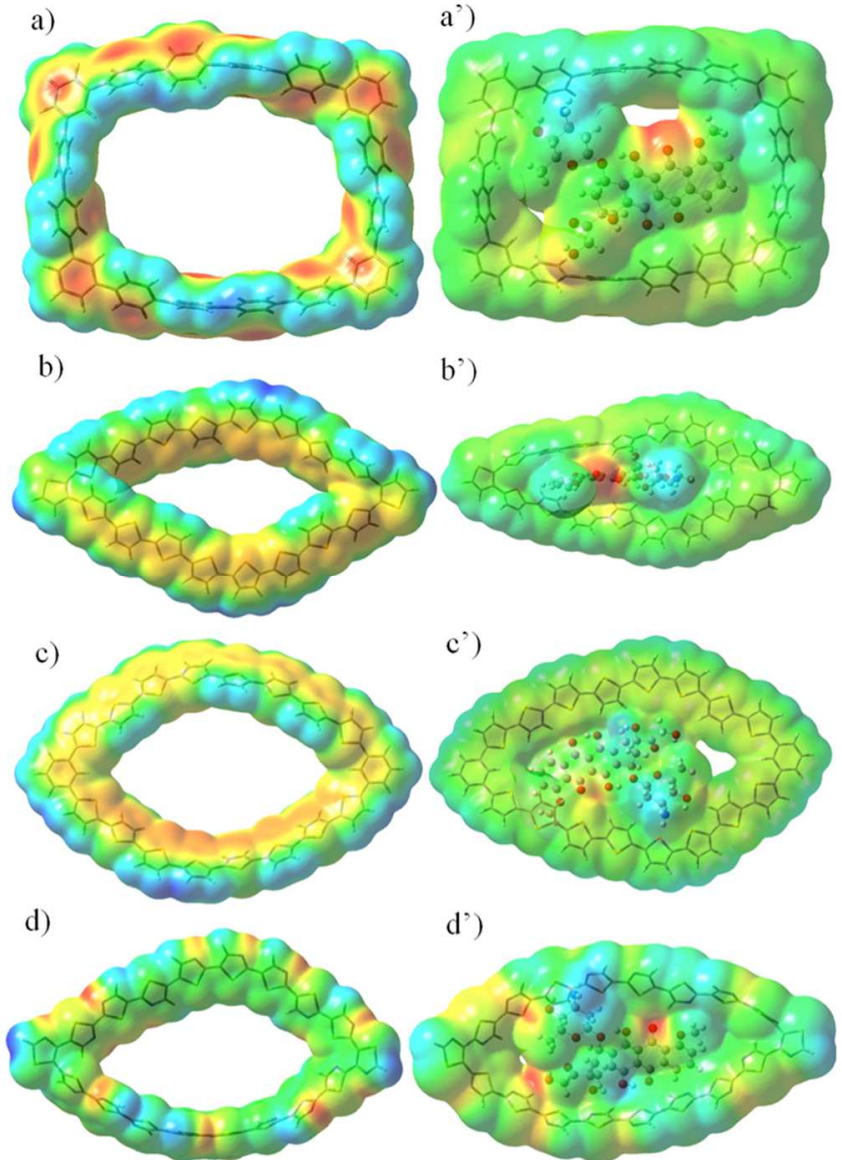

d')

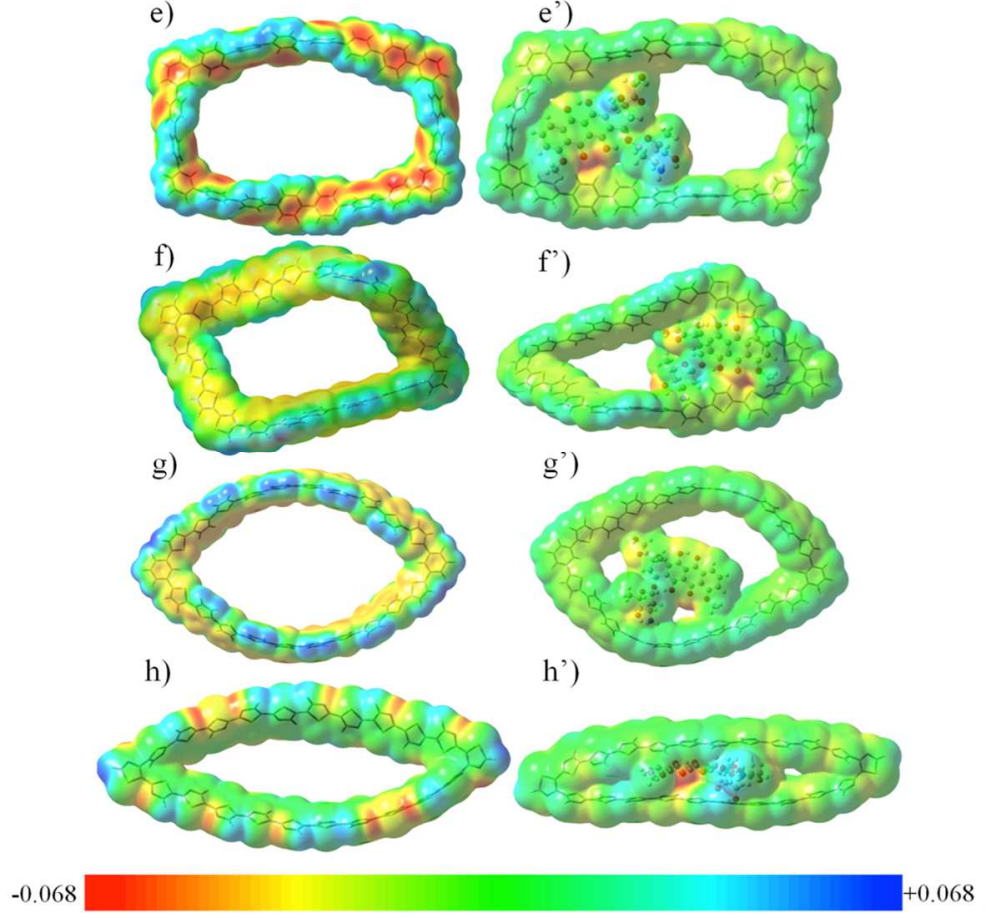

$\left.\mathrm{e}^{\prime}\right)$
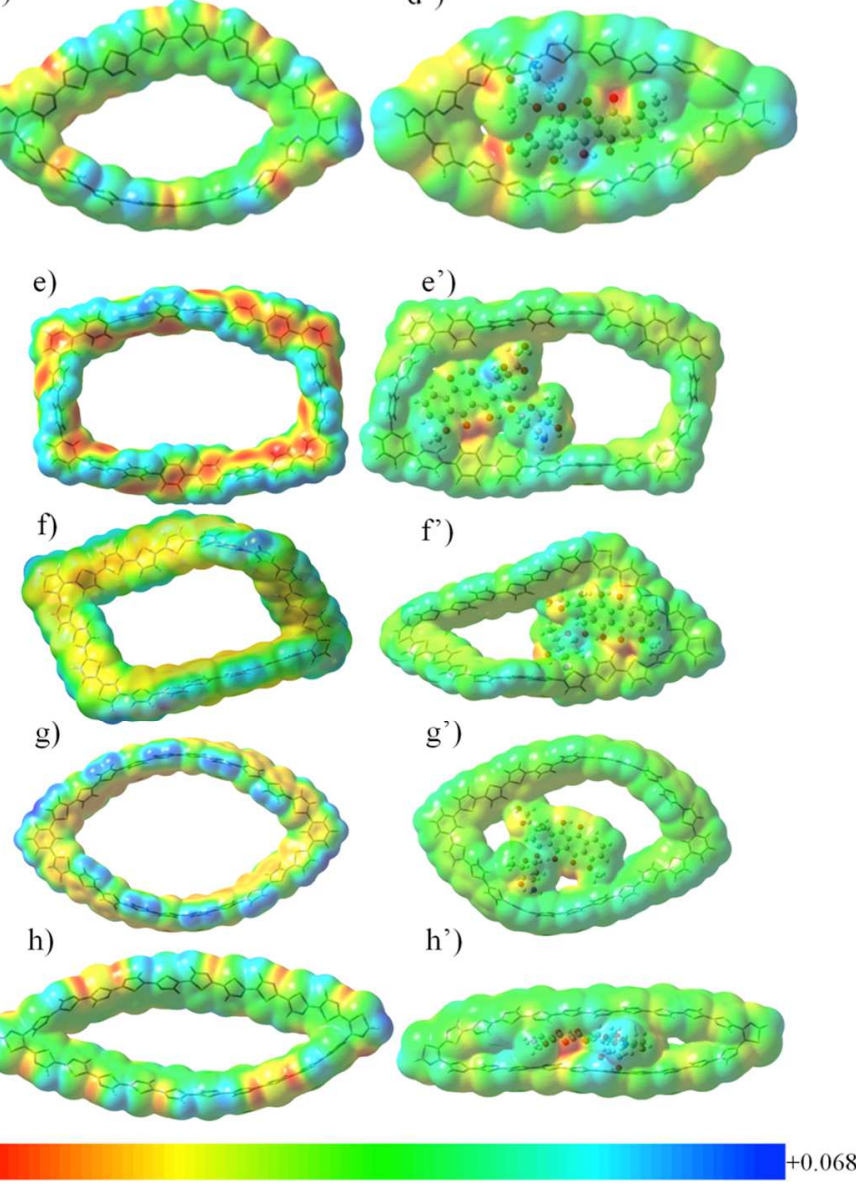

Figure 3: Electrostatic potential (from -0.068 to 0.068 a.u.) of small and large dendrimer cages, with (left) and without (right) encapsulated doxorubicin, mapped over the ground-state electronic isodensity surface (set at 0.02 a.u.). 


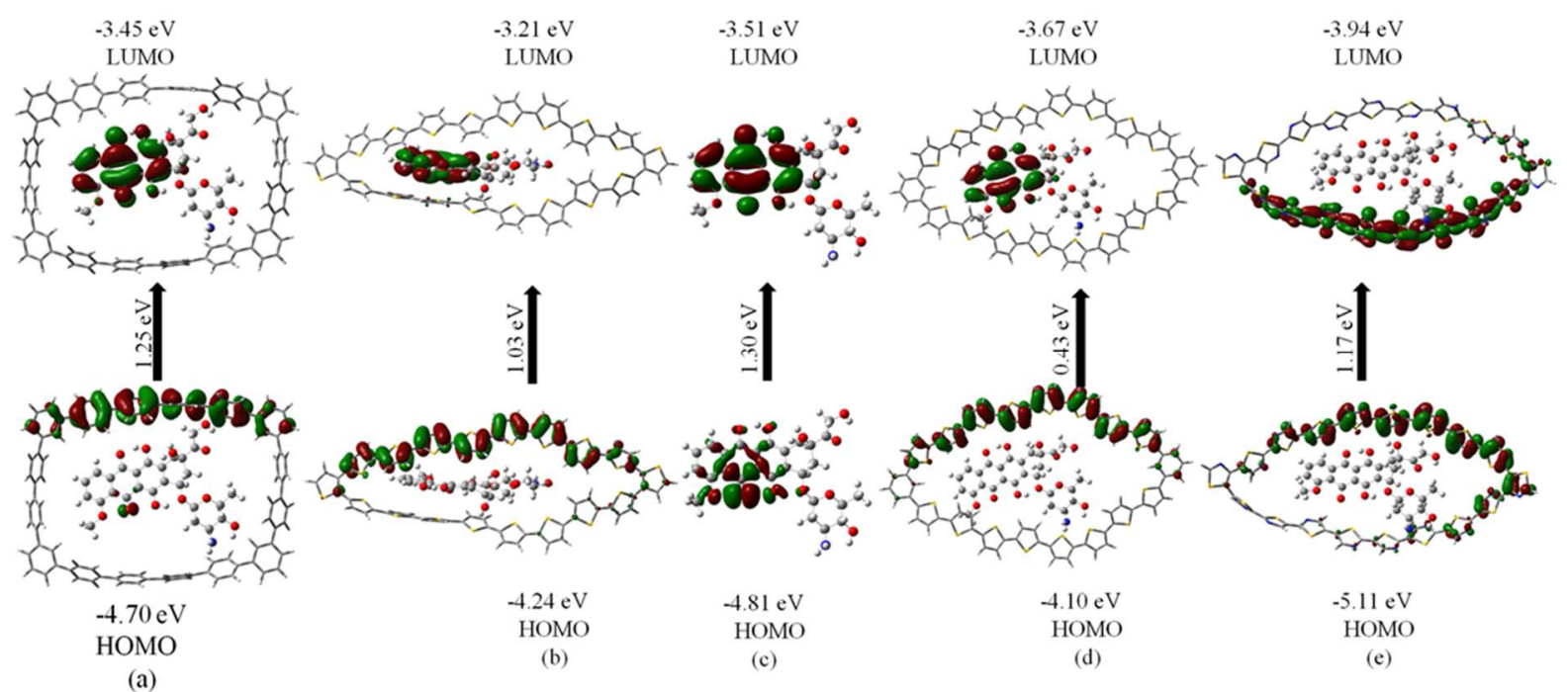

Figure 4: HOMOs and LUMOs, their energies and their energy gaps for (a) DXR@PS, (b) DXR@TS, (c) free DXR, (d) DXR@TPS and (e) DXR@TAS clusters. The isosurfaces are drawn at 0.02 a.u. 


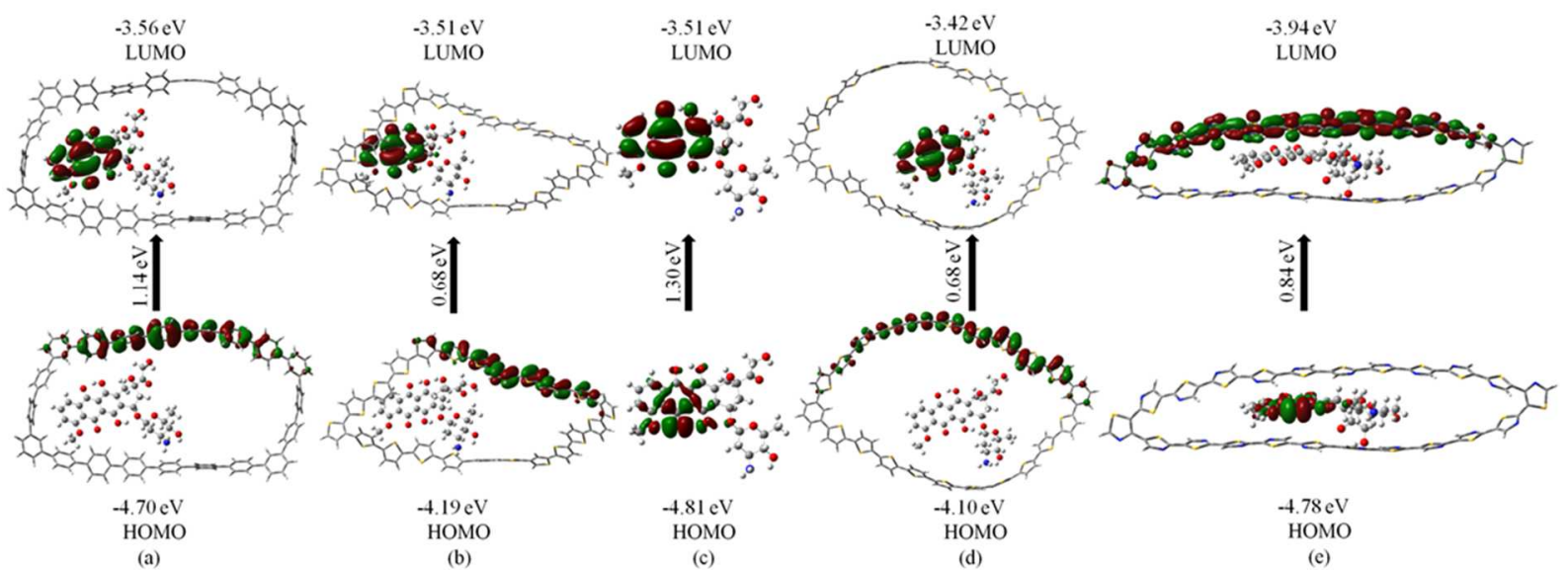

Figure 5: HOMOs and LUMOs, their energies and their energy gaps for (a) DXR@PL, (b) DXR@TL, (c) free DXR, (d) DXR@TPL and (e) DXR@TAL. The isosurfaces are drawn at 0.02 a.u. 